\title{
Prospects for non-standard Higgs Bosons at the LHC
}

\author{
Elizabeth Locci* \\ CEA, DAPNIA / Service de Physique des Particules, CE-Saclay \\ F-91191 Gif-sur-Yvette, France \\ E-mail: elizabeth.locci@cern.ch
}

ABSTRACT: The Large Hadron Collider (LHC) at CERN will provide proton-proton collisions at a centre-of-mass energy of $14 \mathrm{TeV}$ with a design luminosity of $10^{34} \mathrm{~cm}^{-2} \mathrm{~s}^{-1}$. The present report will give a non-exhaustive panorama of the several search methods for the Higgs sector that have been elaborated by the ATLAS and CMS Collaborations

\section{Introduction}

The Minimal Supersymmetric extensions of the Standard Model predict two complex scalar field doublets, with a total of eight degrees of freeedom. As in the Standard Model, three of them are associated to the longitudinal modes of the $\mathrm{W}^{ \pm}$and $\mathrm{Z}^{0}$ bosons. The remaining five degrees of freedom are manifested in five physical scalar Higgs states $\mathrm{h}^{0}, \mathrm{~A}^{0}, \mathrm{H}^{0}$, and the charged Higgs bosons $\mathrm{H}^{ \pm}$. Results are expressed in terms of two parameters which are generally chosen as $\mathrm{m}_{\mathrm{A}}$ and $\tan \beta$, the ratio of vacuum expectation values of the two Higgs fields.

At the tree level, the mass of the lighter of the two CP-even Higgs bosons is limited to be less than the mass of the $\mathrm{Z}^{0}$. Radiative corrections, particularly from loops containing the top quark, allow the lightest Higgs boson mass limit to go up to $\approx 135 \mathrm{GeV} / \mathrm{c}^{2}[1]$ The masses of the other four Higgs bosons are less constrained, but should essentially be degenerate once their mass is larger than $\approx 200 \mathrm{GeV} / \mathrm{c}^{2}$.

The relative importance of the different production modes varies with $\tan \beta$. As low values of $\tan \beta$ have been largely exluded by LEP data, we will mainly concentrate on the large $\tan \beta$ scenario where the associated production mode $\mathrm{b} \overline{\mathrm{b}} \mathrm{H}_{\mathrm{SUSY}}$ is predominant. Over much of the parameter space considered, the neutral Higgs bosons decay predominantly to $\mathrm{b} \overline{\mathrm{b}}$ and $\tau^{+} \tau^{-}$, and the charged Higgs mainly decay to bt and $\tau \nu_{\tau}$. At relatively large masses $\left(\mathrm{m}_{\mathrm{H} \text { SUSY }} \geq 300 \mathrm{GeV} / \mathrm{c}^{2}\right)$, decay modes involving neutralinos or charginos may become important. These modes will not be discussed here, but ATLAS and CMS studies give promising perspectives.

${ }^{*}$ Speaker. 

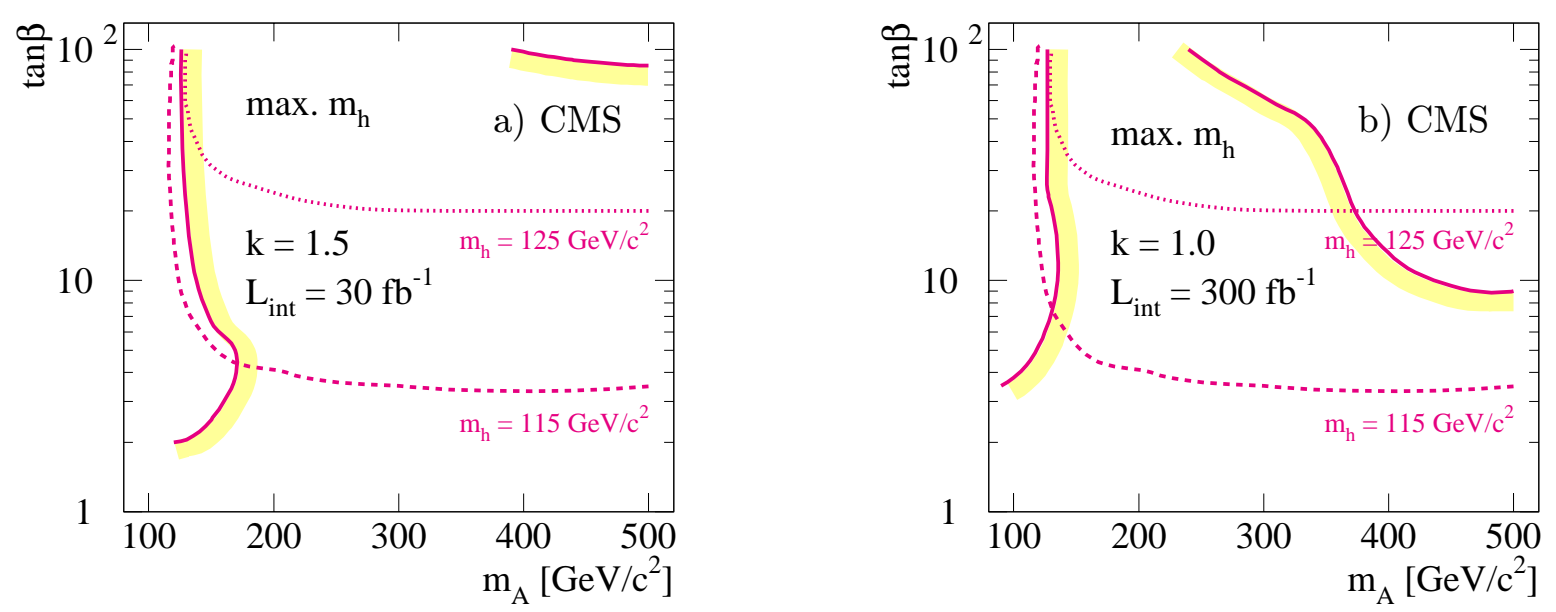

Figure 1: a) Discovery contours in the MSSM ( $\mathrm{m}_{\mathrm{h}^{0}}$-max scenario) parameter space for $30 \mathrm{fb}^{-1}$, using $t \bar{t} h^{0}$ associated production. $\mathrm{S} / \sqrt{\mathrm{B}} \geq 5$ to the right side of the solid line. The dotted and dashed lines are the isomass curves for $\mathrm{m}_{\mathrm{h}^{0}}=125 \mathrm{GeV} / \mathrm{c}^{2}$ and $\mathrm{m}_{\mathrm{h}^{0}}=115 \mathrm{GeV} / \mathrm{c}^{2}$. b) Discovery contours in the MSSM ( $\mathrm{m}_{\mathrm{h}^{0}}$-max scenario) parameter space for $300 \mathrm{fb}^{-1}$, using $\mathrm{W}^{ \pm} \mathrm{h}^{0}$ associated production. $\mathrm{S} / \sqrt{\mathrm{B}} \geq 5$ to the right side of the solid line. The dotted and dashed lines are the isomass curves for $\mathrm{m}_{\mathrm{h}^{0}}=125 \mathrm{GeV} / \mathrm{c}^{2}$ and $\mathrm{m}_{\mathrm{h}^{0}}=115 \mathrm{GeV} / \mathrm{c}^{2}$. In this scenario, $\mathrm{M}_{\mathrm{SUSY}}=1 \mathrm{TeV} / \mathrm{c}^{2}$, $\mu=0.2 \mathrm{TeV} / \mathrm{c}^{2}, \mathrm{M}_{2}=0.2 \mathrm{TeV} / \mathrm{c}^{2}, \mathrm{M}_{\tilde{\mathrm{g}}}=0.8 \mathrm{M}_{\mathrm{SUSY}}, \mathrm{X}_{\mathrm{t}}^{\mathrm{OS}}=2 \mathrm{M}_{\mathrm{SUSY}}, \mathrm{X}_{\mathrm{t}}^{\overline{\mathrm{MS}}}=\sqrt{6} \mathrm{M}_{\mathrm{SUSY}}$,

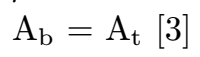

\section{Search methods}

\subsection{Neutral Higgs bosons}

At masses above the range explored by LEP, the dominant decay of the neutral Higgs bosons is to $b \bar{b}$. This mode is essentially impossible to separate from the huge b $\bar{b}$ background from QCD in case of inclusive $h^{0}$ production. However, a signal can be extracted when the Higgs boson is produced in association with a $t \bar{t}$ pair or a $\mathrm{W}^{ \pm}\left[\overline{2} \bar{i}\right.$. In $\mathrm{t}_{\mathrm{t}} \mathrm{h}^{0}$ associated production, using a full simulation of the physics processes and of the detector response, a signal significance ${ }^{1}$ larger than 5 can be reached with $30 \mathrm{fb}^{-1}$ for $\mathrm{m}_{\mathrm{h}^{0}} \leq 125 \mathrm{GeV} / \mathrm{c}^{2}$. The b-tagging performances (efficiency and purity) are crucial and the shape of the background must be measured from data. A good understanding of the jet-jet effective mass signal shape and of the background is essential. In $\mathrm{W}^{ \pm} \mathrm{h}^{0}$ associated production, preliminary studies show that a signal significance of 6 can be obtained, requiring however $\approx 300 \mathrm{fb}^{-1}$, and full background subtraction is necessary to give evidence for a signal. Figure i'i, shows the reach of the $\mathrm{h}^{0} \rightarrow \mathrm{b} \overline{\mathrm{b}}$ channel in the MSSM parameter space in the $\mathrm{m}_{\mathrm{h}^{0}}$-max scenario, the most unfavourable case as signal visibility decreases with increasing mass. These measurements will also provide information on th and WWh couplings with a precision of $\approx 10 \%$.

The most interesting decay channels of the heavy neutral Higgs bosons $\left(\mathrm{A}^{0}, \mathrm{H}^{0}\right)$ are $\tau^{+} \tau^{-}$and $\mu^{+} \mu^{-}$, as b $\bar{b}$ decays are essentially unseparable from the huge QCD b $\bar{b}$ background. In the $\tau^{+} \tau^{-}$channel, three final states can be used: e $\mu$, lepton- $\tau$ jet, 2 hadrons (from $\tau$ jets) with opposite charges [i] tection of the signal at large $\tan \beta$ where the $\mathrm{b} \bar{b} H_{\text {SUSY }}$ production mechanism dominates.

\footnotetext{
${ }^{1}$ The signal significance is defined as $\mathrm{S} / \sqrt{\mathrm{B}}$, where $\mathrm{S}$ is the signal and $\mathrm{B}$ is the background
} 


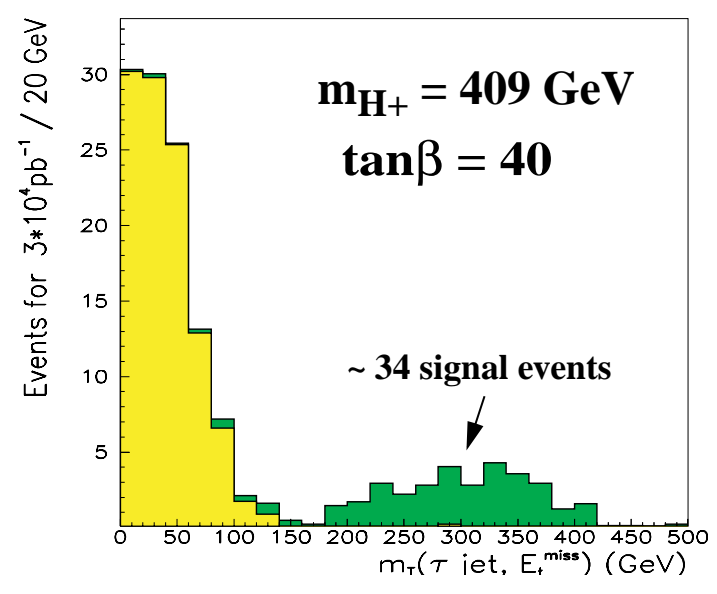

Figure 2: The transverse mass distribution of signal and backgrounds for a charged Higgs search using $30 \mathrm{fb}^{-1}$. The couplings are determined in the MSSM with $\mathrm{m}_{\mathrm{H}^{ \pm}}=409 \mathrm{GeV} / \mathrm{c}^{2}$ and $\left.\tan \beta=40[]_{[-1}^{\overline{4}}\right]$

Despite the 2 (or more) neutrinos from $\tau$ decays escaping detection, a $\tau^{+} \tau^{-}$mass reconstruction is possible. Mass resolutions of 12 to $16 \%$ can be obtained in the various $\tau^{+} \tau^{-}$ final states. The $2 \tau$ jets final state provides the best mass resolution. The main difficulty in this channel is triggering in the presence of the large QCD background, however successful trigger strategies have been elaborated by CMS. An interesting new development is the use of $\tau$-tagging by impact parameter measurements on one-prong leptonic or hadronic $\tau$ decays which are possible thanks to the expected impact parameter resolutions of $\approx 20-30 \mu \mathrm{m}$ in the LHC pixel-trackers.

\subsection{Charged Higgs bosons}

A charged Higgs boson with a mass larger than the top mass will predominantly decay to bt, but this channel is highly contaminated by the QCD background. The $\mathrm{H}^{ \pm} \rightarrow \tau^{ \pm} \nu_{\tau}$

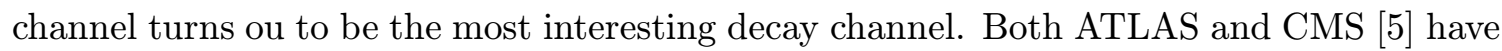
studied the production mechanism $\mathrm{pp} \rightarrow \mathrm{H}^{ \pm} \mathrm{tb}+X$, followed by the decay $\mathrm{H}^{ \pm} \rightarrow \tau^{ \pm} \nu_{\tau}$. The $\tau$-polarization effects can be exploited to enhance the signal over the background. Due to the scalar nature of the decaying $\mathrm{H}^{+}$, the $\tau^{+}$from the $\mathrm{H}^{+}$decay is produced in a left-handed polarization state, whilst, due to the vector nature of the $\mathrm{W}^{+}$, the $\tau^{+}$from the $\mathrm{W}^{+}$decay is produced left-handed, when the $\mathrm{W}$ originates from $\mathrm{t} \overline{\mathrm{t}}$ which is the main background. Thus harder pions (from $\tau \rightarrow \pi \nu$ ) are expected from $\mathrm{H}^{ \pm}$decays than from $\mathrm{W}^{ \pm}$decays to taus. To improve the signal to background ratio, the $\mathrm{W}$ and top masses are reconstructed from jets, requiring one tagged b-jet. In the purely hadronic final states an almost background-free signal is found in the transverse mass distribution reconstructed from the $\tau$-jet and the missing transverse energy (Figure $\overline{2}$ il).

\section{MSSM Higgs parameter space coverage}

All studies of the MSSM Higgs sector, including some which have not been discussed here, are summarized in terms of $\left(\mathrm{m}_{\mathrm{A}}, \tan \beta\right)$ parameter space coverage for a $5 \sigma$ discovery 

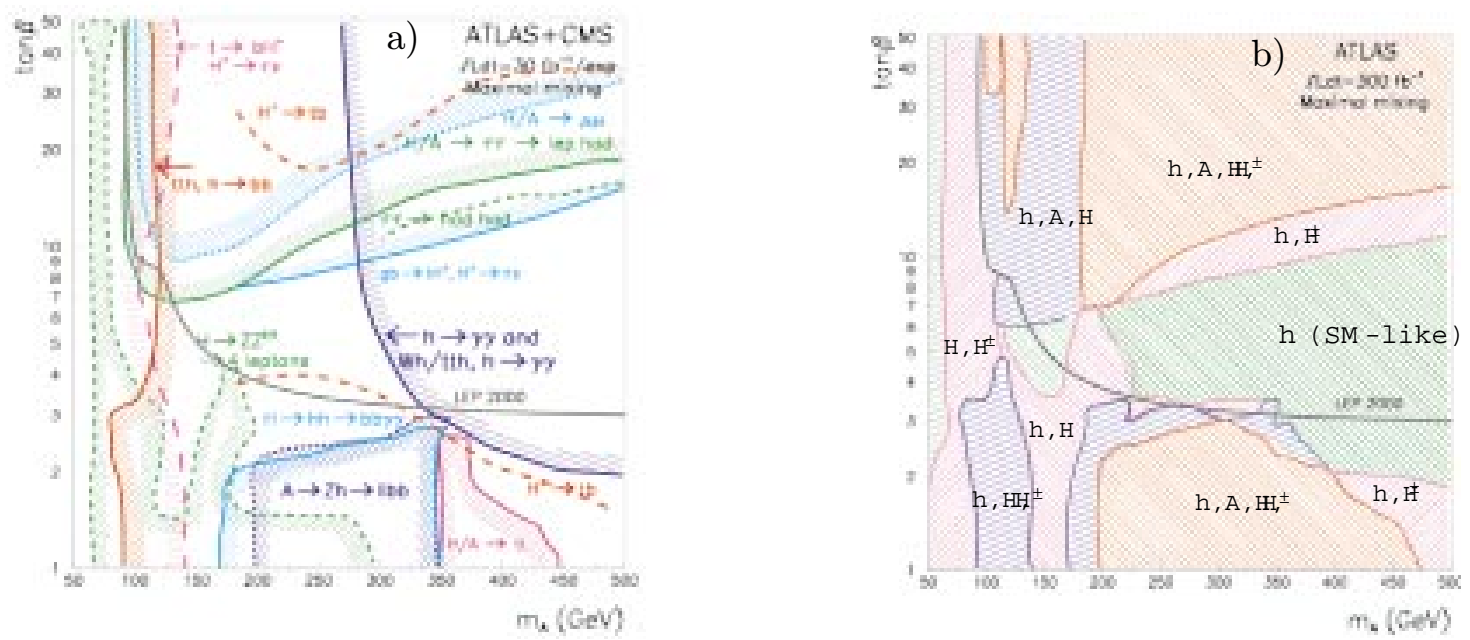

Figure 3: a) $5 \sigma$ discovery contours in the $\left(\mathrm{m}_{\mathrm{A}}, \tan \beta\right)$ plane for $\mathrm{m}_{\mathrm{t}}=175 \mathrm{GeV} / \mathrm{c}^{2}$, $\mathrm{M}_{\mathrm{SUSY}}=1 \mathrm{TeV} / \mathrm{c}^{2}$ and maximal mixing, combining ATLAS and CMS with $30 \mathrm{fb}^{-1}$ per experiment. Not all channels are shown and SUSY particles are assumed to be heavy. b) $5 \sigma$ discovery contours in the $\left(\mathrm{m}_{\mathrm{A}}, \tan \beta\right)$ plane for $1,2,3$, and 4 Higgs bosons using $300 \mathrm{fb}^{-1}$ for ATLAS alone.

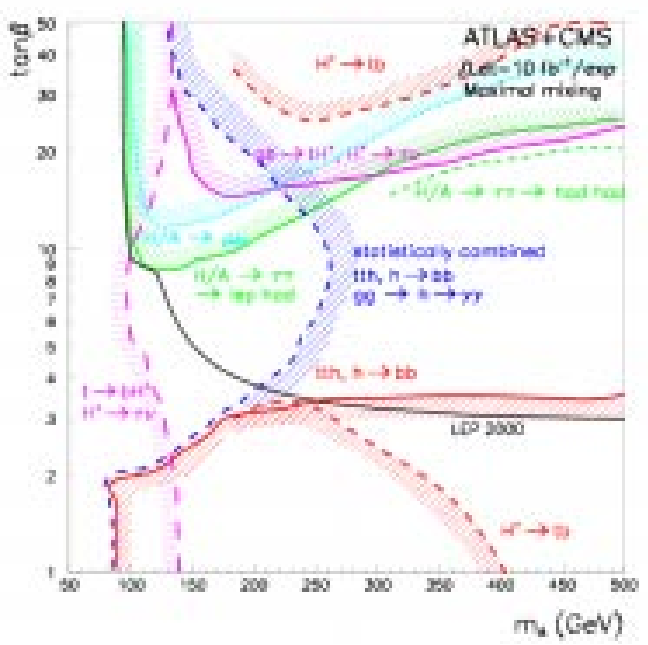

Figure 4: $5 \sigma$ discovery contours in the $\left(\mathrm{m}_{\mathrm{A}}, \tan \beta\right)$ plane for $\mathrm{m}_{\mathrm{t}}=175 \mathrm{GeV} / \mathrm{c}^{2}, \mathrm{M}_{\mathrm{SUSY}}=1 \mathrm{TeV} / \mathrm{c}^{2}$ and maximal mixing, combining ATLAS and CMS with $10 \mathrm{fb}^{-1}$ per experiment.

in Figure $\overline{3}$ a. This figure shows the $5 \sigma$ discovery contours in the $\left(\mathrm{m}_{\mathrm{A}}, \tan \beta\right)$ plane for $\mathrm{m}_{\mathrm{t}}=175 \mathrm{GeV} / \mathrm{c}^{2}, \mathrm{M}_{\mathrm{SUSY}}=1 \mathrm{TeV} / \mathrm{c}^{2}$, and maximal stop-mixing ${ }^{2}$. By combining the results of the two experiments, the plane is expected to be fully covered already with $30 \mathrm{fb}^{-1}$. Two or more Higgs bosons can be observed with $300 \mathrm{fb}^{-1}$ over most of the parameter space, allowing to differentiate between the observation of a SM Higgs from that of a MSSM Higgs (Figure $\bar{\beta} \mathrm{b} b$ ). However there remains a region at $\mathrm{m}_{\mathrm{A}} \geq 200 \mathrm{GeV} / \mathrm{c}^{2}$ and $4 \geq \tan \beta \leq 10$ where only the $\mathrm{h}^{0}$ would be seen and it would be indistinguishable

\footnotetext{
${ }^{2}$ for minimal mixing, the range below $115 \mathrm{GeV} / \mathrm{c}^{2}$ has been fully explored by LEP.
} 
from a SM Higgs. Even if only $10 \mathrm{fb}^{-1}$ are delivered to each experiment during the first year of running, Figure $\mathrm{si}_{1}^{\mathrm{i}}$ shows that, nonetheless, a large part of the plane is expected to be explored with ATLAS and CMS combined data.

\section{Conclusions}

LHC has a large discovery potential for MSSM Higgs bosons. The MSSM Higgs sector can be fully explored, and two or more Higgs bosons should be observable over large portions of parameter space allowing for precise measurements of their parameters (masses, couplings, $\tan \beta$ ). New promising channels, such as Higgs decays to sparticles, are under investigation. Calculations are continuously improved to better understand the physics reach and to define the trigger strategies. ATLAS and CMS are actively preparing for discovery of the Higgs bosons and the measurement of their properties.

\section{Acknowledgments}

I wish to express my gratitude to my colleagues for their help in the preparation of this contribution, and in particular: Fabiola Gianotti and Karl Jakobs from ATLAS, Daniel Denegri, Volker Drollinger, Ritva Kinnunen, and Alexandre Nikitenko from CMS.

\section{References}

[1] S. Heinemeyer, W. Hollik and G. Weiglein, Phys.Rev. D58 (1998) 091701, Eur.Phys. J. C9 (1999) 343, Phys. Lett. B440 (1998) 296, hep-ph/9807423 and JHEP 0006 (2000) 009.

[2] ATLAS Collaboration, ATLAS detector and Physics Performance Technical Design Report, CERN/LHCC/99-14, http://atlasinfo.cern.ch/Atlas/GROUPS/PHYSICS/TDR/access.html.

V. Drollinger, Reconstruction and analysis methods for searches of Higgs bosons in the decay mode $\mathrm{h}^{0} \rightarrow \mathrm{b} \overline{\mathrm{b}}$ at hadron colliders, $\mathrm{PhD}$ Thesis, Karlsruhe, July 2001.

[3] M. Carena, S. Heinemeyer, C.E.M. Wagner and G. Weiglein, hep-ph/9912223.

[4] S. Lehti, R. Kinnunen and J. Tuominiemi, CMS Note 1998/019; R. Kinnunen and A. Nikitenko, CMS Note 1997/106; R. Kinnunen and D. Denegri, CMS Note 1999/037.

D. Cavalli, et al., ATLAS Note PHYS-NO-025 (1993).

[5] R. Kinnunen, CMS Note 2000/045.

K.A. Assamagan and Y. Coadou, ATL-PHYS-2000-031. 